Probability, Networks and Algorithms

Probability, Networks and Algorithms
PNA A note on pooling of labels in random fields

M.N.M. van Lieshout, R.S. Stoica

Report PNA-E0906 June 2009 
Centrum Wiskunde \& Informatica (CWI) is the national research institute for Mathematics and Computer Science. It is sponsored by the Netherlands Organisation for Scientific Research (NWO).

$\mathrm{CWI}$ is a founding member of ERCIM, the European Research Consortium for Informatics and Mathematics.

CWI's research has a theme-oriented structure and is grouped into four clusters. Listed below are the names of the clusters and in parentheses their acronyms.

\section{Probability, Networks and Algorithms (PNA)}

Software Engineering (SEN)

Modelling, Analysis and Simulation (MAS)

Information Systems (INS)

Copyright (C) 2009, Centrum Wiskunde \& Informatica

P.O. Box 94079, 1090 GB Amsterdam (NL)

Science Park 123, 1098 XG Amsterdam (NL)

Telephone +31 205929333

Telefax +31 205924199

ISSN 1386-3711 


\section{A note on pooling of labels in random fields}

\section{ABSTRACT}

This paper studies the effect on the interaction structure of merging labels in random field models.

2000 Mathematics Subject Classification: 60G60

Keywords and Phrases: Label merging; Markov connected component field; Markov random field. 



\title{
A Note on Pooling of Labels in Random Fields
}

\author{
M.N.M. van Lieshout \\ $C W I$ \\ P.O. Box 94079, 1090 GB Amsterdam, The Netherlands \\ Department of Mathematics and Computer Science, Eindhoven University of Technology \\ P.O. Box 513, 5600 MB Eindhoven, The Netherlands \\ R.S. Stoica \\ Paul Painlevé Laboratory, University of Lille 1 \\ 59655 Villeneuve d'Ascq Cedex, France
}

\begin{abstract}
This paper studies the effect on the interaction structure of merging labels in random field models.

2000 Mathematics Subject Classification: 60G60

Keywords and Phrases: Label merging, Markov connected component field, Markov random field.
\end{abstract}

\section{Introduction}

In image segmentation, often Markov random field models are employed in which neighbouring (blocks) of pixels have a similar texture [11]. Generally, the number of different textures is unknown. To overcome this problem, a number of Bayesian approaches have been suggested in which the unknown number is treated as a random variable. See for example $[4,7]$.

A practical problem with such approaches is that most Markov random field densities are known only up to a normalising constant. When updating the number of labels in a Monte Carlo method, the normalising constants do not cancel out and have to be approximated. A more fundamental problem is that the interaction structure may change dramatically if two labels are pooled together, in other words, the class of Markov random fields is not closed under merging labels, making them unnatural models in an unsupervised image segmentation algorithm. In contrast, we show that the class of Markov connected component models [10] is closed under the above mentioned operation, and hence may provide more natural prior distributions for image segmentation with an unknown number of different textures. 
The plan of this note is as follows. First we review some random field theory in Section 2, the main Section 3 studies the effect on the interaction structure of changes in the number of labels and compares the results to their counterparts in a continuous point process set-up $[2,9]$. The paper closes with a short discussion.

\section{Markov and connected component fields}

Let $S=\left(s_{1}, \ldots, s_{m}\right)$ be a collection of sites, for example a rectangle of raster points in $\mathbb{Z}^{2}$, and assume there is a symmetric, reflexive relation $\sim$ on $S$. In a graph theoretical interpretation, the sites are the vertices, and an edge is drawn between $s$ and $r, s \neq r$, if and only if $s \sim r$. Each site is assigned a label, or colour, from the set $\Lambda=\{1, \ldots, q\}$, $q \geq 2$, at random according to some probability distribution. The labels are nominal, so any convenient set of $q$ distinct numbers may be used. The induced random field is denoted by $X=\left(X_{1}, \ldots, X_{m}\right)$ with $X_{i}$ indicating the label at $s_{i}$.

There is a close connection between the notion of a random field and the physics concept of a Gibbs state. Recall that $X$ is a Gibbs state with interaction potentials $\left\{V_{A}: A \subseteq S\right\}$, if

$$
P\left(X_{1}=x_{1}, \ldots, X_{m}=x_{m}\right)=\frac{1}{Z} \exp \left[\sum_{A \subseteq S} V_{A}\left(x_{1}, \ldots, x_{m}\right)\right]
$$

for $V_{A}: \Lambda^{S} \rightarrow \mathbb{R}$ such that $V_{\emptyset}(\cdot) \equiv 0$ and $V_{A}(\cdot)$ depends only on the sites in $A$. The potential $V$ is normalised with respect to the label $\ell \in \Lambda$ if $x_{a}=\ell$ for some $a \in A$ implies $V_{A}\left(x_{1}, \ldots, x_{m}\right)=0$. In fact, any random field with strictly positive probability mass function is a Gibbs state with respect to the $\ell$-canonical potential

$$
V_{A}\left(x_{1}, \ldots, x_{m}\right)=\sum_{B \subseteq A}(-1)^{|A \backslash B|} \log P\left(\mathbf{x}^{B}\right)
$$

where $\mathbf{x}_{s}^{B}=x_{s}$ for $s \in B$ and a prefixed value $\ell \in \Lambda$ otherwise. This is the unique normalised solution with respect to label $\ell$. See [5] for proofs and further details. From now on, we shall assume that $P(\cdot)$ is strictly positive so that conditional distributions are well-defined, and use the equivalent Gibbs state formulation ad libitum.

A random field $X$ with probability mass function $P(\cdot)$ is said to be Markov with respect to $\sim$ if for all $i=1, \ldots, m$ the conditional probability mass function

$$
P\left(X_{i}=x_{i} \mid X_{j}=x_{j}, j \neq i\right)=P\left(X_{i}=x_{i} \mid X_{j}=x_{j}, s_{j} \sim s_{i}, j \neq i\right)
$$

depends only on $x_{i}$ and the labels at those sites $s_{j}, j \neq i$, that share an edge with $s_{i}$ $[5,11]$. The collection $\partial\left(s_{i}\right)$ of such sites is called the neighbourhood of the $i^{\text {th }}$ site; the conditional distributions in (2) are known as the local characteristics. 
To characterise Markov random fields, we need the following definition. A clique is a subset $C \subseteq S$ for which $s \sim t$ for all $s, t \in C$. Note that singletons and the empty set $\emptyset$ are cliques. Write $\mathcal{C}$ for the class of all cliques in $S$. Then, the Hammersley-Clifford theorem [3] states that $X$ is a Markov random field if and only if its probability mass function can be written as

$$
P\left(X_{1}=x_{1}, \ldots, X_{m}=x_{m}\right)=\prod_{C \in \mathcal{C}} \varphi_{C}\left(x_{c}, c \in C\right)
$$

for some interaction functions $\varphi_{C}: \Lambda^{C} \rightarrow \mathbb{R}^{+}$defined for each clique $C \in \mathcal{C}$. Equation (3) amounts to saying that $X$ is a Gibbs state with interaction potentials $V_{C}(\cdot)=$ $\log \varphi_{C}(\cdot)$ for non-empty $C$.

The Markov connected component fields proposed by Møller and Waagepetersen [10] are defined by a factorisation of the form

$$
P\left(X_{1}=x_{1}, \ldots, X_{m}=x_{m}\right)=\frac{1}{Z} \prod_{K \in \mathcal{K}(\mathbf{x})} \Psi\left(K, l\left(\mathbf{x}_{K}\right)\right)
$$

where the product ranges over the maximal $\sim$-connected components of identically labelled sites in $\mathbf{x}=\mathbf{x}^{S}=\left(x_{1}, \ldots, x_{m}\right), l\left(\mathbf{x}_{K}\right)$ denotes the common label in the component $\mathbf{x}_{K}=\left(x_{k}, k \in K\right)$, and $\Psi(\cdot, \cdot)$ is a positive function on $\mathcal{K} \times \Lambda$, the product space of all $\sim$-connected components and the label set. In general, a local dependence definition does not exist, except when one of the colours may be regarded as background.

It is important to observe that the two classes are not comparable in the sense that neither class is contained in the other, see [10] for further details.

\subsection{Example: the Potts model}

Let $S=\left(s_{1}, \ldots, s_{m}\right)$ be a finite set of sites, $\sim$ a symmetric, reflexive relation on $S$ and $\Lambda=\{1, \ldots, q\}, q \geq 2$, a finite set of labels. Then the Potts model is a $\Lambda^{S}$-valued random variable with joint probability mass function

$$
\pi\left(x_{1}, \ldots, x_{m}\right)=\frac{1}{Z} \exp \left[-\beta \sum_{s_{i} \sim s_{j}, i<j} 1\left\{x_{i} \neq x_{j}\right\}\right] .
$$

The parameter $\beta \in \mathbb{R}$ is known as the reciprocal temperature, $Z$ is the normalising constant that ensures $\sum_{\mathbf{x} \in \Lambda^{S}} \pi(\mathbf{x})=1$. The special case $q=2$ is known as the Ising model. Thus, (5) is of the form (1) with $V_{C}\left(x_{i}, x_{j}\right)=-\beta 1\left\{x_{i} \neq x_{j}\right\}$ for the two-point set $C=\left\{s_{i}, s_{j}\right\}$ with $s_{i} \sim s_{j}$, and $V_{A} \equiv 0$ otherwise.

The Potts model (5) has local characteristics given by

$$
\frac{P\left(X_{i}=\ell \mid X_{j}=x_{j}, j \neq i\right)}{P\left(X_{i}=1 \mid X_{j}=x_{j}, j \neq i\right)}=\frac{\exp \left[-\beta \sum_{s_{i} \sim s_{j}} \mathbf{1}\left\{x_{j} \neq \ell\right\}\right]}{\exp \left[-\beta \sum_{s_{i} \sim s_{j}} 1\left\{x_{j} \neq 1\right\}\right]}
$$


for any $\ell \in \Lambda$ and $x_{j} \in \Lambda, j \neq i \in\{1, \ldots, m\}$. We conclude that $X$ is Markov with respect to $\sim$ for all $q$. If $\beta>0$, majority voting amongst the neighbours of the site of interest determines which label has the highest conditional probability (ferromagnetic case); for $\beta<0$, the label disagreeing with most of the neighbours' is most likely (anti-ferromagnetic case). The interaction functions are $\varphi_{\emptyset}=1 / Z$, $\varphi_{C}\left(x_{i}, x_{j}\right)=\exp \left[-\beta 1\left\{x_{i} \neq x_{j}\right\}\right]$ for cliques $C=\left\{s_{i}, s_{j}\right\}$ that consist of a pair of neighbours $s_{i} \sim s_{j}$, and $\varphi_{C} \equiv 1$ otherwise.

Since

$$
\pi\left(x_{1}, \ldots, x_{m}\right)=\frac{1}{Z} \exp \left[-\frac{\beta}{2} \sum_{K \in \mathcal{K}(\mathbf{x})} \sum_{s_{i} \in K}\left|\left\{s_{j} \notin K: s_{i} \sim s_{j}\right\}\right|\right]
$$

the Potts model is also a Markov connected component field with respect to $\sim$.

\section{Merging of labels}

Chin and Baddeley [2] proved that the class of Markov connected component point processes [1] is closed under independent superposition. The purpose of the present paper is to show that the same is true for Markov connected component fields under merging of labels.

Theorem 1. Let $S=\left(s_{1}, \ldots, s_{m}\right)$ be a finite set of sites, $\sim$ a symmetric, reflexive relation on $S$ and $\Lambda=\{1, \ldots, q\}, q \geq 2$, a finite set of labels. Let $X$ be a q-colour Markov connected component field (4) with respect to $\sim$ and define the random field $Y$ with values in $\{0, \ldots, q-2\}$ by

$$
Y_{i}=X_{i} \mathbf{1}\left\{X_{i} \leq q-2\right\}
$$

for $i=1, \ldots, m$. Then $Y$ is a $(q-1)$-colour Markov connected component field with respect to $\sim$.

Proof: $\quad$ Fix $\mathbf{y}=\left(y_{1}, \ldots, y_{m}\right) \in\{0, \ldots, q-2\}^{S}$. For $\mathbf{x} \in \Omega_{\mathbf{y}}:=\left\{\mathbf{x} \in \Lambda^{S}: y_{i}=\right.$ $\left.x_{i} \mathbf{1}\left\{x_{i} \leq q-2\right\}, i=1, \ldots, m\right\}$ and $j \in \Lambda$, write $\mathcal{K}_{j}(\mathbf{x})$ for the set of maximal connected components in $\mathbf{x}$ labelled $j$. Note that the maximal $j$-labelled connected components in $\mathbf{x}$ and $\mathbf{y}$ are identical for $j=1, \ldots, q-2$, and that each $(q-1)$ - or $q$-component is part of a single maximal 0 -component in $\mathbf{y}$. Denote the family of these 0 -components by $\mathcal{K}_{0}(\mathbf{y})$. Now, the probability mass function of $Y$ is given by

$$
\pi_{Y}(\mathbf{y})=\sum_{\mathbf{x} \in \Omega_{\mathbf{y}}} \pi(\mathbf{x})=\frac{1}{Z} \prod_{j=1}^{q-2} \prod_{K \in \mathcal{K}_{j}(\mathbf{y})} \Psi(K, j) \times \prod_{K \in \mathcal{K}_{0}(\mathbf{y})}\left\{\sum_{\mathbf{x} \in\{q-1, q\}^{K}} \prod_{j=q-1}^{q} \prod_{L \in K_{j}(\mathbf{x})} \Psi(L, j)\right\}
$$


which proves the claim.

Note that the normalisation constant $Z$ involved in $\pi_{Y}$ is the same as that in $\pi$, the probability mass function of $X$.

The factorisation in the proof above should be compared to that for point processes, see $[2]$ or $[8$, p. 73$]$.

A similar result does not hold for Markov random fields. Here the situation is more complicated, reflecting the state of affairs for Markov point processes with respect to superposition [8, 9].

Example 1. Consider the Potts model $X$ introduced in Section 2.1, and, for $i=$ $1, \ldots, m$, set $Y_{i}=X_{i} \mathbf{1}\left\{X_{i} \leq q-2\right\}$. Then the probability mass function of $Y$ equals

$$
\begin{aligned}
\pi_{Y}(\mathbf{y}) & =\sum_{\mathbf{x} \in \Omega_{\mathbf{y}}} \frac{1}{Z} \exp \left[-\beta \sum_{s_{i} \sim s_{j}, i<j} 1\left\{x_{i} \neq x_{j}\right\}\right]=\frac{1}{Z} \exp \left[-\beta \sum_{s_{i} \sim s_{j}, i<j} 1\left\{y_{i} \neq y_{j}\right\}\right] \\
& \times \prod_{K \in \mathcal{K}_{0}(\mathbf{y})}\left\{\sum_{\mathbf{x} \in\{q-1, q\}^{K}} \exp \left[-\beta \sum_{s_{i} \sim s_{j} \text { in } K, i<j} \mathbf{1}\left\{x_{i} \neq x_{j}\right\}\right]\right\} .
\end{aligned}
$$

Thus, (6) is proportional to the product of the probability mass functions of a Potts model with $q-1$ colours and a Markov connected component field on the maximal 0components collected in $\mathcal{K}_{0}(\mathbf{y})$. Realisations of $X$ and $Y$ with $q=5$ are shown in the two left-most panels of Figure 1.

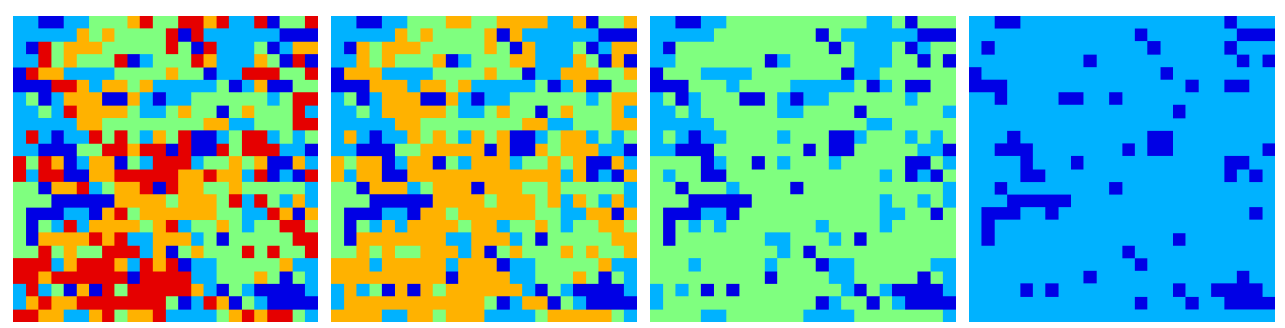

Figure 1: Realisation of a Potts model with $q=5$ labels, the first order neighbour relation $s_{i} \sim s_{j} \Leftrightarrow\left\|s_{i}-s_{j}\right\| \leq 1$, and interaction parameter $\beta=1$ (left-most panel) after merging two labels successively (other panels).

We conclude that merging two labels in a Potts model yields a Markov connected component field with respect to the underlying relation $\sim$. One might wonder whether $Y$ is Markov with respect to $\sim$. This is not the case, as can be seen from the following counterexample. 
Counterexample 1. Consider the following configuration with sites indexed in row major order and first order neighbour relation $s_{i} \sim s_{j} \Leftrightarrow\left\|s_{i}-s_{j}\right\| \leq 1, i=1, \ldots, 4$

$0 ?$

$0 / 10$

Let $X$ be a Potts model with $q=3$ and $\beta=1$, and merge labels 2 and 3 into a single label 0 to obtain the random field $Y$. We are interested in the marginal distribution of $Y$ at the top right site given its neighbours are assigned value 0 . Straightforward computations yield $P\left(Y_{2}=1 ; Y_{1}=Y_{3}=Y_{4}=0\right)=2 e^{-2}\left(1+e^{-1}\right)^{2} / Z=P\left(Y_{1}=Y_{2}=\right.$ $\left.Y_{4}=0 ; Y_{3}=1\right)$. Also $P\left(Y_{1}=Y_{2}=Y_{3}=Y_{4}=0\right)=\left(2+2 e^{-4}+12 e^{-2}\right) / Z$, and $P\left(Y_{1}=Y_{4}=0 ; Y_{2}=Y_{3}=1\right)=4 e^{-4} / Z$. We obtain

$$
P\left(Y_{2}=1 \mid Y_{1}=Y_{3}=Y_{4}=0\right)=\frac{e^{-2}\left(1+e^{-1}\right)^{2}}{1+7 e^{-2}+2 e^{-3}+2 e^{-4}},
$$

respectively

$$
P\left(Y_{2}=1 \mid Y_{1}=Y_{4}=0 ; Y_{3}=1\right)=\frac{2 e^{-2}}{1+2 e^{-1}+3 e^{-2}} .
$$

Hence $Y$ is not first-order Markov.

In the above counterexample, the neighbours of the pixel of interest were labelled 0 . To see why, consider any $q$-colour Markov random field $X$ with respect to some relation $\sim$, and focus (without loss of generality) on the site $s_{1}$. Let $\left(y_{2}, \ldots, y_{m}\right)$ be a configuration on the remaining sites such that the labels fall in $\{1, \ldots, q-2\}$ on the neighbourhood $\partial\left(s_{1}\right)$ of $s_{1}$. Define $Y$ as in Theorem 1 . Then for $\ell \in\{1, \ldots, q-2\}$,

$$
\begin{aligned}
P\left(Y_{1}=\ell, Y_{j}=y_{j}, j \neq 1\right) & =\sum_{\mathbf{x} \in \Omega_{\mathbf{y}}} P\left(X_{1}=l, X_{j}=x_{j}, j \neq 1\right) \\
& =\sum_{\mathbf{x} \in \Omega_{\mathbf{y}}} P\left(X_{1}=\ell \mid X_{j}=x_{j}, s_{j} \in \partial\left(s_{1}\right)\right) P\left(X_{j}=x_{j}, j \neq 1\right) \\
& =P\left(Y_{1}=\ell \mid Y_{j}=y_{j}, s_{j} \in \partial\left(s_{1}\right)\right) P\left(Y_{j}=y_{j}, j \neq 1\right)
\end{aligned}
$$

where as before $\Omega_{\mathbf{y}}=\left\{\mathbf{x} \in \Lambda^{S}: y_{i}=x_{i} \mathbf{1}\left\{x_{i} \leq q-2\right\}\right\}$. Hence

$$
P\left(Y_{1}=\ell \mid Y_{j}=y_{j}, j \neq 1\right)=P\left(Y_{1}=\ell \mid Y_{j}=y_{j}, s_{j} \in \partial\left(s_{1}\right)\right)
$$

for any $\ell \neq 0$. Since probabilities add to unity, also

$$
P\left(Y_{1}=0 \mid Y_{j}=y_{j}, j \neq 1\right)=P\left(Y_{1}=0 \mid Y_{j}=y_{j}, s_{j} \in \partial\left(s_{1}\right)\right) .
$$

For homogeneous models such as the Potts model in Counterexample 1, a single neighbour of the pixel of interest may be set to zero without affecting the relation (2) 
for $Y$. A similar phenomenon may is observed for Markov point processes, that are invariant under independent superposition up to second order [8, p. 59-61].

Next, we turn our attention to the class of random fields that are both Markov and connected component Markov, characterised by Møller and Waagepetersen [10] by a factorisation of the probability mass function as follows:

$$
\pi\left(x_{1}, \ldots, x_{m}\right)=\frac{1}{Z} \prod_{K \in \mathcal{K}(\mathbf{x})} \prod_{\emptyset \neq C \in \mathcal{C}, C \subseteq K} \Phi\left(C, l\left(\mathbf{x}_{K}\right)\right) .
$$

For instance for the Potts model introduced in Subsection 5 , for $C=\{c\}$ with $c \in K$, $\Phi\left(C, l\left(\mathbf{x}_{K}\right)\right)=\exp \left[-\frac{\beta}{2}\left|\left\{s_{j} \notin K: c \sim s_{j}\right\}\right|\right]$ with $\Phi \equiv 1$ otherwise.

We have the following corollary to Theorem 1.

Corollary 1. Let $S=\left(s_{1}, \ldots, s_{m}\right)$ be a finite set of sites, $\sim$ a symmetric, reflexive relation on $S$ and $\Lambda=\{1, \ldots, q\}, q \geq 2$, a finite set of labels. Let $X$ be a q-colour Markov connected component field with respect to $\sim$ with a probability mass function given by (7) and define the random field $Y$ with values in $\{0, \ldots, q-2\}$ by

$$
Y_{i}=X_{i} \mathbf{1}\left\{X_{i} \leq q-2\right\}
$$

for $i=1, \ldots, m$. Then $Y$ is a $(q-1)$-colour Markov connected component field with respect to $\sim$ with

$$
\Psi(K, 0)=\sum_{\mathbf{x} \in\{q-1, q\}^{K}} \prod_{j=q-1}^{q} \prod_{L \in K_{j}(\mathbf{x})} \prod_{\emptyset \neq C \in \mathcal{C}, C \subseteq L} \Phi(C, j) .
$$

In general, $Y$ is not a Markov random field, see Counterexample 1).

For any Markov random field, the following result holds true.

Theorem 2. Let $S=\left(s_{1}, \ldots, s_{m}\right)$ be a finite set of sites, $\sim$ a symmetric, reflexive relation on $S$ and $\Lambda=\{1, \ldots, q\}, q>2$, a finite set of labels. Let $X$ be a Markov random field (3) with respect to $\sim$ and define the random field $Y$ with values in $\{0, \ldots, q-2\}$ by

$$
Y_{i}=X_{i} 1\left\{X_{i} \leq q-2\right\}
$$

for $i=1, \ldots, m$. Then $Y$ has probability mass function $\pi_{Y}(\mathbf{y})$ given by

$\prod_{\mathcal{C} \ni C \subseteq\left(\cup \mathcal{K}_{0}(\mathbf{y})\right)^{c}} \varphi_{C}\left(y_{c}, c \in C\right) \prod_{K \in \mathcal{K}_{0}(\mathbf{y})}\left\{\sum_{\mathbf{x} \in\{q-1, q\}^{K}} \prod_{\mathcal{C} \ni C \cap K \neq \emptyset} \varphi_{C}\left(\mathbf{x}_{c}, c \in C \cap K ; y_{d}, d \in C \backslash K\right)\right\}$. 
Proof: $\quad$ Fix $\mathbf{y}=\left(y_{1}, \ldots, y_{m}\right) \in\{0, \ldots, q-2\}^{S}$, and write $\Omega_{\mathbf{y}}:=\left\{\mathbf{x} \in \Lambda^{S}: y_{i}=\right.$ $\left.x_{i} \mathbf{1}\left\{x_{i} \leq q-2\right\}, i=1, \ldots, m\right\}$. Then the probability mass function of $Y$ is given by

$$
\begin{aligned}
\pi_{Y}(\mathbf{y}) & =\sum_{\mathbf{x} \in \Omega_{\mathbf{y}}} \pi(\mathbf{x})=\prod_{\mathcal{C} \ni C \subset\left(\cup \mathcal{K}_{0}\right)^{c}} \varphi_{C}\left(y_{c}, c \in C\right) \times \\
& \times \sum_{\mathbf{x} \in\{q-1, q\}^{\mathcal{K}_{0}(\mathbf{y})}} \prod_{\mathcal{C} \ni C \cap \mathcal{K}_{0}(\mathbf{y}) \neq \emptyset} \varphi_{C}\left(\mathbf{x}_{c}, c \in C \cap \mathcal{K}_{0}(\mathbf{y}) ; y_{d}, d \in C \backslash \mathcal{K}_{0}(\mathbf{y})\right) .
\end{aligned}
$$

The key observation is that if $y_{i}=0=y_{j}$ and $s_{i} \sim s_{j}$ then $s_{i}$ and $s_{j}$ must belong to the same maximal 0 -component in $\mathbf{y}$, so no clique can contain points from disjoint $K, L \in \mathcal{K}_{0}(\mathbf{y})$. Consequently, $\pi_{Y}$ is as claimed.

We conclude that $Y$ is not necessarily a Markov connected component field. A counterexample is the Geman and Reynolds field [6]. More precisely, let $S=\left\{s_{1}, s_{2}\right\}$ consist of two related sites, and take $q=6$. Define a random field $X$ by its probability mass function $\pi\left(x_{1}, x_{2}\right) \propto \exp \left[1 /\left(\left|x_{1}-x_{2}\right|+1\right)\right]$. Then clearly $X$ is a Markov random field with

$$
\phi_{S}\left(x_{1}, x_{2}\right)=\exp \left[1 /\left(\left|x_{1}-x_{2}\right|+1\right)\right],
$$

$\phi_{s_{1}}\left(x_{1}\right)=\phi_{s_{2}}\left(x_{2}\right)=1$, and the interaction function of the empty set equal to the normalising constant. However, by arguments similar to those in [10, Ex. A2], the density of $Y$ does not factorise over the maximal connected components.

\section{Conclusion}

In this paper, we considered the effect of merging labels on the interaction structure of random fields. It was shown that the class of Markov connected component fields is closed under the merge operation, and explicit expressions were derived for the component potentials. We then proved that the class of Markov random fields is not closed under the merge operation, indeed the resulting random field may not satisfy the Markov connected component condition.

Our results suggest that models such as those proposed in [10] are natural prior distributions for image segmentation with an unknown number of different textures. Except for trivial cases, Monte Carlo methods will be needed so that the practitioner must be able to jump between $X$ and $Y$. Given $Y=\mathbf{y}$, there are many valid choices to assign label $q$ or $q-1$ to the zero-components of $\mathbf{y}$, for example picking them independently and uniformly. Alternatively, and more naturally, one could sample from the conditional distribution $P(X=\mathbf{x} \mid Y=\mathbf{y})=\pi(\mathbf{x}) 1\left\{\mathbf{x} \in \Omega_{\mathbf{y}}\right\} / \pi_{Y}(\mathbf{y})$. For a Markov connected component field,

$$
P(X=\mathbf{x} \mid Y=\mathbf{y})=\prod_{K \in \mathcal{K}_{0}(\mathbf{y})}\left\{\frac{\prod_{j=q-1}^{q} \prod_{K \in K_{j}(\mathbf{x})} \Psi(K, j)}{\sum_{\mathbf{z} \in\{q-1, q\}^{K}} \prod_{j=q-1}^{q} \prod_{L \in K_{j}(\mathbf{z})} \Psi(L, j)}\right\}
$$


by the proof of Theorem 1, so the $\mathbf{x}$-labels in different maximal 0-components of $\mathbf{y}$ are assigned independently. Switching between labels this way is amenable to Gibbs sampling $[5,11]$ and involves no ratio of normalising constants. Instead, a sample from (8) is required. Since single texture components tend be small compared to the image size, and only two labels have to be considered, such label assignment is quite feasible. 


\section{References}

[1] A.J. Baddeley and J. Møller. Nearest-neighbour Markov point processes and random sets. International Statistical Review, 57:89-121, 1989.

[2] Y.C. Chin and A.J. Baddeley. On connected component Markov point processes. Advances in Applied Probability (SGSA), 31:279-282, 1999.

[3] P. Clifford. Markov random fields in statistics. In Disorder in physical systems. A volume in honour of J.M. Hammersley, G.R. Grimmett and D.J.A. Welsh, eds. Clarendon Press, Oxford, 1990.

[4] I.L. Dryden, M.R. Scarr, and C.C. Taylor. Bayesian texture segmentation of weed and crop images using reversible jump Markov chain Monte Carlo methods. Applied Statistics, 52:31-50, 2003.

[5] D. Geman. École d'été de probabilités de Saint-Flour XVIII - 1988, volume 1427 of Lecture Notes in Mathematics, chapter Random fields and inverse problems in imaging. Springer-Verlag, Berlin, 1990.

[6] D. Geman and G. Reynolds. Constrained restoration and the recovery of discontinuities. IEEE Transactions on Pattern Analysis and Machine Intelligence, 14:367-383, 1992.

[7] P.J. Green and S. Richardson. Hidden Markov models and disease mapping. Journal of the American Statistical Association, 97:1055-1070, 2002.

[8] M.N.M. van Lieshout. Markov point processes and their applications. Imperial College Press/World Scientific, London/Singapore, 2000.

[9] M.N.M. van Lieshout. Propagation of spatial interaction under superposition. In Accuracy 2000, Proceedings of the 4th international symposium on spatial accuracy assessment in natural resources and environmental sciences, G.B.M. Heuvelink and M.J.P.M. Lemmens, eds. Delft University Press, 2000.

[10] J. Møller and R.P. Waagepetersen. Markov connected component fields. Advances in Applied Probability (SGSA), 30:1-35, 1998.

[11] G. Winkler. Image analysis, random fields, and Markov chain Monte Carlo methods: A mathematical introduction. Springer-Verlag, Berlin, Second Edition, 2003. 